\title{
L'économie mondialisée de Robert Reich : approche géographique
}

\section{Gilles Ardinat}

\section{(2) OpenEdition}

\section{Journals}

Édition électronique

URL : http://journals.openedition.org/cdg/1935

DOI : $10.4000 /$ cdg. 1935

ISSN : 2107-7266

Éditeur

UMR 245 - CESSMA

\section{Référence électronique}

Gilles Ardinat, «L'économie mondialisée de Robert Reich : approche géographique », Carnets de géographes [En ligne], 1 | 2010, mis en ligne le 01 octobre 2010, consulté le 23 septembre 2020. URL http://journals.openedition.org/cdg/1935; DOI : https://doi.org/10.4000/cdg.1935

Ce document a été généré automatiquement le 23 septembre 2020

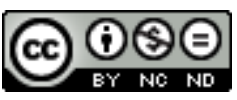

La revue Carnets de géographes est mise à disposition selon les termes de la Licence Creative Commons Attribution - Pas d'Utilisation Commerciale - Pas de Modification 4.0 International. 


\title{
L'économie mondialisée de Robert Reich : approche géographique
}

\author{
Gilles Ardinat
}

1 Les liens entre la géographie et l'économie sont nombreux et anciens. Certains économistes ont apporté une contribution notable à la géographie (Johann Heinrich von Thünen (1826), Alfred Marshall (1890), Alfred Weber (1909) ou August Lösch (1940)). Pour autant, ces deux disciplines, loin de fusionner, conservent leur identité et peuvent aboutir à des conclusions divergentes. Depuis deux décennies, la manière dont est traité le thème de la mondialisation offre une bonne illustration de ces spécificités disciplinaires.

2 Sur ces questions, il est intéressant de consulter le best-seller de Robert Reich: L'Économie mondialisée. En effet, M. Reich, professeur à l'Université de Berkeley, a été très influent sur le plan politique, puisqu'il fut Secrétaire d'État au travail lors du premier mandat de Bill Clinton. Ses analyses de la mondialisation ont fait date dans la littérature économique. En dépit de la notoriété mondiale de cet ouvrage, qui est construit autour de concepts qui intéressent la géographie (mondialisation, distance, frontière, réseau...), il est intéressant de noter que ses principales conclusions sont rejetées par la plupart des géographes français.

\section{L'obsolescence du paradigme national}

L'idée centrale de l'ouvrage de Robert Reich est que la mondialisation a transformé chaque économie nationale (ou « économie-nation » présentée dans la première partie [pp. 23-67]) en un simple sous-ensemble d'une économie devenue planétaire.

4 Reich reconnaît qu'au début du XXe siècle (marqué par une production de masse standardisée de type fordiste), il existait un «compromis national », c'est-à-dire la convergence des intérêts des États, des citoyens et des grandes firmes (qui fournissaient emplois et produits de consommation). Ce compromis national rend pertinents les concepts d'économie nationale et de patriotisme économique. Or, cet accord tacite entre acteurs nationaux perd son sens dans l'économie actuelle, marquée 
par une émancipation des firmes par rapport à leur espace d'origine : «Les firmes et les investisseurs parcourent désormais le monde à la recherche des meilleurs opportunités de profit. Ils se sont déconnectés de leur propre nation » [p.18].

\section{L'entreprise-réseau : un concept organisationnel dont la dimension spatiale est négligée}

D'après la seconde partie de L'Économie mondialisée, le mode de production industrielle a évolué au cours des dernières décennies. De nombreuses firmes se sont détournées d'une "production de masse standardisée » de type pyramidal et centralisé au profit d'une "production personnalisée", beaucoup plus rémunératrice (fortes valeurs ajoutées) et mobilisant des salariés beaucoup plus qualifiés, autonomes et réactifs face aux besoins des clients. Ces salariés innovants sont appelés «manipulateurs de symboles» par Reich. Cette analyse reprend l'idée de l'émergence d'une économie post-fordiste. Ainsi, «de nouveaux réseaux structurent l'entreprise de production personnalisée, et remplacent les vieilles pyramides de l'entreprise de production de masse ; ils sont en train de s'étendre sur l'ensemble du globe. De ce fait, il n'y aura plus de firme américaine, britannique, française, japonaise ou allemande » [p.101].

Reich évoque l'idée d'une dispersion géographique de la production à travers le monde. Alors que la notion traditionnelle du territoire (la nation dans les termes de l'auteur) est remise en cause par les pratiques spatiales des firmes transnationales, les salariés qui forment l'élite économique (les manipulateurs de symboles) se sentent appartenir à un réseau mondial et leur allégeance nationale est affaiblie. Bien que ne faisant l'objet d'aucune tentative de spatialisation systématique (l'ouvrage ne comporte aucune carte), cette théorie oppose, sans les nommer, les logiques territoriales (nations) et réticulaires (les acteurs de la mondialisation). Ces sujets, traités de manière allusive par Reich, ont depuis étés développés par des géographes français, notamment Bertrand Badie (1995) qui oppose les logiques de réseaux et de territoires, mais aussi Pierre Veltz (1996) qui articule mode de production post- fordiste, éclatement géographique des firmes et mondialisation ou enfin Olivier Dollfus (1997) avec son concept d'archipel mondial.

\section{Les risques de " sécession » induits par les mutations de l'économie}

7 Reich ébauche dans sa troisième partie une nouvelle segmentation de la population active en grands types d'emplois. Considérant les découpages traditionnels comme obsolètes, il évoque quatre catégories. Un quart de la population active est relativement protégée (agriculture, extraction et fonction publique). Un autre quart est soumis à la concurrence féroce des pays émergents (les travailleurs routiniers, assurant «les services de production courante »). Ils apparaissent comme les grands perdants de la mondialisation. Ensuite viennent les fournisseurs de «services personnels» (soit $30 \%$ de la population active). Ils sont relativement précaires, mais moins directement menacés par les délocalisations. Enfin, $20 \%$ de la population bénéficie de la mondialisation. Il s'agit des manipulateurs de symboles (avocats d'affaire, traders, 
ingénieurs, créatifs, publicitaires...). Leurs revenus sont de plus en plus décalés par rapport au reste de la population.

8 Cette nouvelle sociologie du travail, essentiellement centrée sur les États-Unis, fait craindre à Reich une "sécession des manipulateurs de symboles » [p.259] vis-à-vis des classes lésées par la mondialisation. Un certain égoïsme fiscal menace le financement des solidarités nationales. De plus, des ségrégations apparaissent à l'intérieur des territoires (enclaves urbaines, banlieues résidentielles sécurisées, communautés fermées...). Ces fractures socio- spatiales fragilisent la cohésion territoriale. Reich se demande donc: «Formons-nous encore une communauté, bien que nous ne soyons plus une économie? » [p.18]. Formulée dans les termes de la politique française, cela revient à s'interroger sur notre identité économique nationale.

\section{Quelles préconisations face aux dangers de la mondialisation?}

9 Compte tenu des inégalités croissantes et du risque de désagrégation sociale, Reich prône un «nationalisme économique positif » [p.295]. Cette politique doit redonner de la vigueur au lien national sans pour autant se couper du reste du monde. L'auteur préconise un plan massif d'investissement dans la formation des travailleurs les plus pénalisés par la mondialisation. Cette qualification doit atténuer les effets de la concurrence et "engendrer de nouvelles efficacités »[p.234], tout en favorisant la mobilité sociale. Un tel programme doit être financé par une fiscalité plus importante sur les revenus des manipulateurs de symboles. Ainsi, la solidarité fiscale et l'amélioration $\mathrm{du}$ facteur travail doivent rendre les nations plus unies et plus compétitives.

10 En outre, cette méthode, qui rejette le protectionnisme, doit favoriser un état d'harmonie, et non de guerre commerciale, avec les autres pays. «Le nationalisme positif tolère aussi, et même favorise, les subventions publiques destinées aux firmes qui mettent en œuvre, à l'intérieur des frontières de la nation, une production à forte valeur ajoutée [...mais] sans aucune distinction fondée sur la nationalité des actionnaires ou celle des dirigeants»[p.297]. Cette préconisation vise à renforcer l'attractivité du territoire national vis-à-vis des investisseurs étrangers.

11 Notons la relative actualité des propositions de Reich. L'idée d'une qualification accrue des travailleurs se retrouve assez largement dans les politiques de compétitivité des territoires (stratégie de Lisbonne, économie de la connaissance) tandis que la recherche des IDE est devenue un élément central des politiques d'attractivité.

\section{Cependant, trois grandes limites peuvent être soulignées}

12 Tout d'abord, il convient de remarquer l'aspect simpliste des solutions apportées par Reich. Lui-même évoque un «catalogue abrégé des moyens d'améliorer la situation difficile des citoyens américains vulnérables dans l'économie mondiale »[p.236]. Ses solutions tiennent, pour l'essentiel en 4 pages [pp. 232-236]. Ce traitement parait rudimentaire. 
13 Ensuite, la nouvelle segmentation de la population active, qui constitue un élément central et innovant, est très peu argumentée. Cette typologie sociale du travail manque cruellement de démonstration et d'explication. Par exemple, les effectifs respectifs de chaque groupe ( $20 \%$ de manipulateurs de symboles, $25 \%$ de travailleurs routiniers...) ne sont appuyés par aucun chiffre précis.

14 Enfin, cet ouvrage, comme souvent en économie, n'a aucune approche multiscalaire. Pour Reich, l'économie est un tout global, de plus en plus intégré : «dans quelques années à peine, il n'y aura virtuellement plus aucun moyen de distinguer une économie nationale d'une autre»[p.158]. Or, cette vision d'un espace unique est largement partielle dans la mesure où la grande majorité des acteurs et des phénomènes humains s'inscrit à d'autres échelles (macro-régionale, nationale ou locale). Cette théorie ne perçoit pas la permanence des ancrages territoriaux. Rappelons qu'à l'échelle mondiale, les filiales étrangères des 82000 firmes transnationales recensées par la CNUCED réalisent moins de $11 \%$ du PIB en 2009 [source : rapport mondial sur l'investissement 2010]. Même aux États-Unis (sujet de prédilection pour Reich), le caractère national de l'économie demeure largement: les exportations de biens et de services ne représentent encore que $12,7 \%$ du PIB [source : OMC 2009], près de 20 ans après la rédaction de L'Économie mondialisée.

\section{Une thèse remise en cause par la plupart des géographes}

Les travaux de Robert Reich défendent, comme ceux de l'économiste japonais Kenichi Ohmae (1989), l'idée selon laquelle la mondialisation serait synonyme de disparition des frontières économiques, de convergence généralisée des modes de vie et d'une uniformisation de la planète. Dans ce monde interconnecté, les entités politiques, au fondement territorial, apparaissent comme dépassées.

Or, les principaux ouvrages français de géographie consacrés à la mondialisation s'attachent au contraire à décrire la mondialisation comme un processus de sélection et de différenciation croissante des territoires. Pour Veltz, « concevoir la globalisation comme l'extension universelle d'un capitalisme homogène est une absurdité » [p.150]. De ce point de vue, les firmes transnationales suivent justement une «stratégie de la maîtrise (et non de la suppression) de la diversité » des territoires [p.124]. En outre, Veltz dénonce le «caractère peu réaliste des images de la firme cosmopolite et anationale » [p.149]. Laurent Carroué (2007), qui considère la mondialisation comme une « valorisation différenciée et sélective des différents territoires par le capital dans un cadre concurrentiel » [p.9], rejette également l'idée d'une économie sans frontière où agissent des firmes apatrides ("World Companies»). Jacques Lévy s'inscrit lui aussi dans une telle vision.

17 L'Économie mondialisée reste pour la géographie un ouvrage de référence dans la mesure où sa thèse centrale suppose une disparition des ancrages nationaux pour les firmes et une disparition de toutes les frontières économiques : c'est contre cette thèse que les géographes se sont mobilisés depuis une quinzaine d'années. Reich n'apporte donc pas de réelle contribution à la réflexion sur les stratégies d'acteurs et sur le rôle des territoires. Le rejet de sa vision peu spatialisée et économiciste constitue une sorte de point de départ aux lectures géographiques de la mondialisation. 


\section{BIBLIOGRAPHIE}

BADIE B., 1995, La Fin des territoires, Paris : Fayard.

CARROUE L., 2007, Géographie de la mondialisation, Paris : Armand Colin DOLLFUS O., 1997, La Mondialisation, Paris : Presse Sciences Po.

OHMAE K., 1989, Managing in a Borderless World, Harvard Business Review, Mai-Juin.

REICH R., 1991, The Work of Nations: Preparing Ourselves for XXIth Century Capitalism, New-York: Alfred A Knopf. Les citations sont tirées de la version française, 1997, L'Économie mondialisée, Paris : Dunod.

VELTZ P., 1996, Mondialisation, villes et territoires : l'économie d'archipel, Paris : Presses Universitaires de France. Les citations sont tirées de l'édition 2005.

INDEX

Thèmes : Carnets de lectures

\section{AUTEUR}

\section{GILLES ARDINAT}

Agrégé ATER EA 3766 GESTER (Gestion des Sociétés, des Territoires et des Risques) Université Paul Valéry-Montpellier III 